\title{
Interaction between polygenic risk for cigarette use and environmental exposures in the Detroit neighborhood health study
}

\author{
JL Meyers ${ }^{1}$, M Cerdá ${ }^{1}$, S Galea ${ }^{1}$, KM Keyes ${ }^{1}$, AE Aiello ${ }^{2}$, M Uddin $^{3,4}$, DE Wildman ${ }^{3}$ and KC Koenen ${ }^{1}$
}

Cigarette smoking is influenced both by genetic and environmental factors. Until this year, all large-scale gene identification studies on smoking were conducted in populations of European ancestry. Consequently, the genetic architecture of smoking is not well described in other populations. Further, despite a rich epidemiologic literature focused on the social determinants of smoking, few studies have examined the moderation of genetic influences (for example, gene-environment interactions) on smoking in African Americans. In the Detroit Neighborhood Health Study (DNHS), a sample of randomly selected majority African American residents of Detroit, we constructed a genetic risk score (GRS), in which we combined top ( $P$-value $<5 \times 10^{-7}$ ) genetic variants from a recent meta-analysis conducted in a large sample of African Americans. Using regression (effective $n=399$ ), we first tested for association between the GRS and cigarettes per day, attempting to replicate the findings from the meta-analysis. Second, we examined interactions with three social contexts that may moderate the genetic association with smoking: traumatic events, neighborhood social cohesion and neighborhood physical disorder. Among individuals who had ever smoked cigarettes, the GRS significantly predicted the number of cigarettes smoked per day and accounted for $\sim 3 \%$ of the overall variance in the trait. Significant interactions were observed between the GRS and number of traumatic events experienced, as well as between the GRS and average neighborhood social cohesion; the association between genetic risk and smoking was greater among individuals who had experienced an increased number of traumatic events in their lifetimes, and diminished among individuals who lived in a neighborhood characterized by greater social cohesion. This study provides support for the utility of the GRS as an alternative approach to replication of common polygenic variation, and in gene-environment interaction, for smoking behaviors. In addition, this study indicates that environmental determinants have the potential to both exacerbate (traumatic events) and diminish (neighborhood social cohesion) genetic influences on smoking behaviors.

Translational Psychiatry (2013) 3, e290; doi:10.1038/tp.2013.63; published online 13 August 2013

\section{Introduction}

Cigarette use is a leading risk factor for multiple illnesses and a major public health concern. ${ }^{1}$ Twin and family studies have demonstrated that smoking is influenced both by genetic and environmental factors. ${ }^{2,3}$ Genome-wide association studies (GWAS) in populations of European ancestry have identified common genetic variation associated with smoking behaviors. An initial GWAS of smoking quantity identified associations with genetic variants in several of the nicotinic acetylcholine receptors clustered on chromosome $15 .{ }^{4}$ Meta-analyses in three large consortia of smoking behaviors confirmed the GWAS finding and refined the association signal within the locus. ${ }^{5,6}$ Additional genetic risk variants have been identified for smoking quantity, including neuronal nicotinic acetylcholine receptor subunit beta-3 (CHRNB3) and alpha-6 (CHRNA6), ${ }^{4}$ cytochrome P450, family 2, subfamily A, polypeptide 6 $(\text { CYP2A6 })^{4,6}$ and LOC10018894, ${ }^{6}$ a variant residing in a gene whose function is currently unknown. Of the variants identified to date, each genetic variant, or single-nucleotide polymorphism (SNP), accounts for a very small proportion of the variance in smoking behavior $(<2 \%)$. Smoking behaviors are complex genetic traits, influenced by multiple genetic variants, ${ }^{7,8}$ which likely interact with the individual's social context. ${ }^{9-12}$ Polygenic risk scores, which aggregate common genetic risk variants, have been increasingly used to model the genetic architecture of complex traits. ${ }^{8,13-15}$ In addition to circumventing the statistical stringency of multiple test corrections necessary for GWAS, this method may more accurately reflect the underlying genetic architecture of complex behaviors, such as cigarette use.

Until last year, all large-scale gene identification studies on smoking were conducted in populations of European ancestry. Conducting genetic association (and gene-environment interaction) studies in populations of African ancestry is important because of their greater genetic diversity and the evolutionary differences in disease allele frequency and linkage disequilibrium patterns. ${ }^{16}$ Due to the lack of gene identification studies in populations of diverse ancestry, the genetic architecture of smoking-related traits is not well described in groups that are not of European ancestry. This

\footnotetext{
${ }^{1}$ Department of Epidemiology, Columbia University, New York, NY, USA; ${ }^{2}$ Department of Epidemiology, University of Michigan, Ann Arbor, MI, USA; ${ }^{3}$ Center for Molecular Medicine and Genetics, Wayne State University, Detroit, MI, USA and ${ }^{4}$ Department of Psychiatry and Behavioral Neurosciences, Wayne State University, Detroit, MI, USA

Correspondence: Dr J Meyers, Department of Epidemiology, Columbia University, 722 West 168th Street, New York, 10032 NY, USA.

E-mail: jlm2261@ columbia.edu

Keywords: African American; cigarette smoking; gene-environment interaction; genetic risk score; neighborhoods; nicotine; traumatic life events

Received 19 March 2013; revised 4 June 2013; accepted 10 July 2013
} 
is an important gap in the literature, as there is evidence that genetic determinants have important implications for multiple addictive behaviors in populations globally. ${ }^{17}$ The Study of Tobacco in Minority Populations (STOMP) Genetics Consortium, which represents 13 GWAS studies of men and women of African ancestry, was created to search for genetic risk loci for smoking behaviors in this population. Recently, STOMP conducted a meta-analysis of smoking phenotypes (for example, number of cigarettes smoked per day and age of smoking initiation) in 32389 African Americans (effective sample size $\sim 15000){ }^{18}$ This well-powered meta-analysis produced one genetic variant of genome-wide significance (cholinergic receptor, nicotinic, alpha 5, CHRNA5 SNP rs2036527, $\left.P<5 \times 10^{-8}\right)$, several additional variants that were approaching significance $\left(P<5 \times 10^{-7}\right)$ and many variants that fell short of statistical significance for the prediction of cigarettes smoked per day.

Whereas the identification of specific genetic variants is necessary to determine the underlying biological mechanism of risk, understanding how these variants interact with aspects of the environment, both on the individual and macro levels, to produce risk for smoking behaviors remains crucial to develop downstream public health interventions. Epidemiologists have long been investigating smoking and related substance-use behaviors in heterogeneous populations. ${ }^{19}$ For example, (self-identified) African Americans, on average, initiate smoking later ${ }^{20}$ and smoke fewer cigarettes per day, ${ }^{20,21}$ yet are less likely to successfully quit smoking. Further, they have a higher risk of smoking-related lung cancer than many other populations. ${ }^{22}$ In addition, individuallevel factors, such as the experience of trauma ${ }^{23}$ and lack of social support from family and peers, ${ }^{24}$ have been associated with increased substance use. ${ }^{25}$

Neighborhood-level factors have also been associated with the risk of substance use. In particular, previous studies have found an inverse relationship between neighborhood social cohesion and substance-use outcomes, ${ }^{26}$ suggesting that neighborhood social cohesion may act as a protective factor against the risk of smoking initiation. In addition, studies that examined the relationship between neighborhood physical characteristics and substance-use outcomes found that poorer neighborhood quality (dilapidated building structures) was associated with substance-use disorders. ${ }^{27,28}$

It is likely that genetic factors and one's social context jointly shape one's risk for smoking behaviors. ${ }^{9-11}$ Further, many of the genetic and environmental risk factors are likely to be shared with other substance-use behaviors and disorders. ${ }^{19,29}$ Although no study to our knowledge has specifically examined the interaction between genetic variants and neighborhood social cohesion or physical disorder, previous studies have reported interactions between genetic variants and individual-level predictors of substance use, such as the experience of traumatic life events. ${ }^{30,31}$ For example, interactions between trauma with genetic variants including dopaminergic and nicotinic receptors have been reported as incurring risk for smoking. ${ }^{32-34}$ Evidence for the interaction between the experience of childhood trauma and risk at the 5HTTLPR locus with regards to smoking behaviors is mixed with both positive ${ }^{35}$ and negative $e^{22,23}$ results. Relatedly, interactions have been observed between aggregated, latent genetic and environmental influences on cigarette smoking using the genetically informative twin-family design. Twin studies have demonstrated that the genetic risk for adolescent smoking is higher in urban than rural communities. ${ }^{36,37}$ The authors posited that urban communities were characterized by decreased community monitoring that allowed for the greater expression of genetic predispositions for adolescent substance use. ${ }^{36,38}$ Taken together, this literature suggests that riskier environments (for example, increased stressful life events and fewer social constraints) enable predispositions towards substance use to be manifested. ${ }^{39}$ Despite this growing GxE literature, few studies have examined the role of gene-environment interactions in African American populations. In addition, previous GxE studies have primarily focused on individual-level factors (the experience of sexual abuse), whereas macro level influences, such as the social environment (neighborhood characteristics) have also been demonstrated to influence smoking behaviors, ${ }^{10,38}$ and may also moderate genetic influences on smoking.

Although investigators have examined gene-environment interactions in smoking using individual genetic variants (candidate genes) or latent genetic influences (twin studies), no study to our knowledge has used polygenic risk scores in the context of GxE for smoking behaviors. A polygenic risk score aggregates measured genetic variants to more closely reflect the underlying structure of complex traits; multiple genetic variants of small effect that act in conjunction with each other (epistasis) and the external environment (GxE). Further, as a polygenic risk score aggregates specific genetic variants, it enables the examination of social factors that may moderate underlying genetic influences. In this study, we expand upon the literature by examining the interaction between an aggregate measure of genetic risk for cigarette smoking and three environmental exposures that have previously been associated with increased substance use: traumatic life events, neighborhood social cohesion and neighborhood physical disorder. In an effort to model the genetic architecture of smoking behaviors in African Americans, the first aim of this study is to use the genetic variants associated ( $P$-value $<5 \times 10^{-7}$ ) with number of cigarettes smoked per day from the STOMP meta-analysis to predict this phenotype in the Detroit Neighborhood Health Study (DNHS), a majority African American population-based sample of individuals living in Detroit. The second aim of this study is to test for interaction between the aggregate measure of molecular genetic risk for cigarette smoking and three environmental exposures: traumatic life events, neighborhood social cohesion and neighborhood physical disorder.

\section{Materials and methods}

Sample. Participants were recruited from the DNHS ( $n=1547$ ), a longitudinal cohort of predominately African American men and women adults $(18+)$ living in Detroit, Michigan using procedures described in more detail elsewhere $^{40}$ (Supplementary Figure 1). Participants were administered a 40-min assessment, which included demographic variables including self-identified race, sex and age, as well as questions on psychopathology, substance use, neighborhood influences and exposure to traumatic events. Assessments 
were administered using structured telephone interviews, and each participant received $\$ 25$ for their participation in the survey. Respondents were also asked to provide blood specimen by way of venipuncture (or by way of saliva when blood was unavailable, $n=125$ ) and received an additional $\$ 25$ if they elected to do so. A total of 778 DNA samples were collected from consenting participants. Of these 778 individuals, ages ranged from 18-95 years (mean $(M)=52.65$, s.d. $=16.38$ ) and $57.7 \%$ were female. When asked how they would describe their racial backgrounds, $82.5 \%$ endorsed Black/African American, with the remainder of the sample endorsing Asian, American Indian or Alaska Native. To reduce stratification, only those who described their backgrounds as African American were included in the present analyses. In addition, only individuals who had smoked cigarettes during their lifetime were included in analyses so that genetic and environmental influences on the decision to initiate cigarette smoking are not confounded with genetic and environmental influences on current frequency of cigarette use.

\section{Measures}

Cigarettes per day. The following question was used to assess the current number of cigarettes smoked each day: In the past 30 days, on those days when you smoked, on average, how many cigarettes did you smoke per day? The number of cigarettes was recorded and standardized (z-score) to create a quantitative measure of cigarettes smoked per day. All subjects were asked whether they had ever smoked cigarettes on at least some days, in their lifetime. Individuals who had never smoked in their lifetime $(41.8 \%)$ were excluded from further analyses.

Traumatic events. The number of traumatic events an individual experienced was assessed using a checklist of 19 items occurring in the individual's entire lifetime including the following: (1) combat or war-zone exposure, (2) rape, (3) sexual assault, (4) shot or stabbed, (5) tortured or kidnapped, (6) mugged or threatened with a weapon, (7) badly beaten, (8) serious motor vehicle accident or injury, (9) serious accident or injury, (10) natural disaster, (11) diagnosed with life-threatening disease, (12) child diagnosed with lifethreatening disease, (13) witnessed murder or serious injury, (14) discovered a dead body, (15) learned that a close friend or relative was raped or sexually assaulted, (16) learned that a close friend or relative was physically attacked, (17) learned that a close friend or relative was seriously injured in a motor vehicle crash, (18) learned that a close friend or relative was injured in any accident and (19) sudden death of a close friend or relative. The number of items endorsed was summed to create a score that could range from 0-19. Higher scores reflect having experienced a greater number of traumatic events. Note that, although throughout this manuscript we refer to these events as traumatic, it is uncertain that these potentially traumatic events induced a negative response in all subjects, merely that the event occurred.

Average neighborhood social cohesion (social cohesion). The following five items probed each individual's perception of the social cohesion in their neighborhood: (1) this is a close-knit or unified community, (2) people around here are willing to help their neighbors, (3) people in this neighborhood generally do not get along with each other (reverse coded), (4) people in this neighborhood do not share the same values (reverse coded) and (5) people in this neighborhood can be trusted. Respondents could agree or disagree with these statements in varying degrees $(1=$ strongly agree through $4=$ strongly disagree). Responses to the items were then summed to create a score that could range from 0 to 16 . Each of these sum scores were then averaged by neighborhood to create a neighborhood-wide measure of social cohesion. Higher scores indicate a greater level of average neighborhood social cohesion. ${ }^{41}$ Note that neighborhoods were defined by geocoding DNHS subjects based on census tracts. ${ }^{28}$ Neighborhood-level perceptions of social cohesion were calculated by aggregating the mean social cohesion scores for all individuals residing in each neighborhood. Participant addresses were geocoded to block groups, which in turn were aggregated to the 54 neighborhoods of Detroit. These neighborhood boundaries are recognized by city planners as established community boundaries. Neighborhood-level social cohesion scores were divided into roughly equal tertiles considering aggregate neighborhood-level social cohesion scores for all the 54 neighborhoods, with a higher score representing greater cohesion. Further details on this construct have been published previously. ${ }^{42}$

Average neighborhood physical disorder (physical disorder). Systematic assessments of neighborhood physical environment were conducted by trained independent observers in a random sample of 138 block groups in all the 54 Detroit neighborhoods. Observers rated the neighborhood physical environment on 19 items adapted from the New York City IMPACT neighborhood evaluation scale. ${ }^{43}$ Factor analyses suggested that three items formed a cohesive measure of the external physical environment, which are as follows: (1) presence of buildings with broken windows; boarded-up windows or boarded-up doors; (2) presence of buildings with outside damage that can only be corrected by major repairs such as siding, shingles, boards, brick, concrete and stucco and (3) presence of entirely vacant buildings. These measures also demonstrated excellent internal consistency $(\alpha=0.83)$. The factor analyses yielded principal component values for each block group, which were detailed previously. ${ }^{28}$ Frequency values were predicted for those areas of Detroit that were not evaluated and maps of these prediction estimates were created. Predicted values were then averaged by neighborhood so that a higher score indicates more physical disorders.

Genome-wide association analysis. DNA was isolated from whole blood $(n=688)$ or, when unavailable, from saliva $(n=125)$. DNA samples were sent to the Applied Genomics Technology Facility (Wayne State University, Detroit, MI, USA) for genotyping using the HumanOmniExpress BeadChips (Illumina, San Diego, CA, USA). Further details regarding genotyping procedures have been published previously. ${ }^{44}$ We genotyped 730525 SNPs. Samples were removed because of low call rate $(<95 \%)$ and duplicate issues, with a remaining sample of 413 women and 365 men. A total of 688890 SNPs passed quality control filters 
Table 1 Descriptive statistics for key variables in the Detroit neighborhood health study

\begin{tabular}{|c|c|c|c|}
\hline \multirow[b]{2}{*}{ Key variable } & \multicolumn{3}{|c|}{ Frequency/mean (s.d.) } \\
\hline & Full sample $\mathrm{n}=1547$ & Full genetic sample $\mathrm{n}=778$ & Analytic sample $\mathrm{n}=399$ \\
\hline Female & $56.9 \%$ & $57.7 \%$ & $51.8 \%^{\mathrm{a}}$ \\
\hline Age & $49.05(17.12)$ & 52.65 (16.38) & $48.29(15.05)$ \\
\hline African American (self-identified) & $86.9 \%$ & $82.5 \%$ & $100 \%^{a}$ \\
\hline Lifetime smoker & $58.2 \%$ & $52.2 \%$ & $100 \%{ }^{a}$ \\
\hline Former smoker & $43.4 \%$ & $44.1 \%$ & $46.5 \%$ \\
\hline Current smoker & $56.6 \%$ & $55.9 \%$ & $53.5 \%$ \\
\hline Cigarettes smoked per day & $11.99(13.27)$ & $11.16(12.19)$ & $11.16(12.19)$ \\
\hline Number of traumatic events & $4.38(3.57)$ & $5.23(3.81)$ & $5.87(3.87)^{a^{\prime}}$ \\
\hline Neighborhood social cohesion & $3.51(1.47)$ & $3.41(1.48)$ & $3.38(1.57)$ \\
\hline Neighborhood physical disorder & $0.14(0.70)$ & $0.10(0.69)$ & $0.15(0.84)$ \\
\hline
\end{tabular}

andicates that the analytic sample significantly differs from the full sample $(P$-value $<0.05)$.

(call rate $>95 \%$, minor allele frequency $>0.01$, HardyWeinberg disequilibrium $P>1 \times 10^{-6}$ ). Genotype reports generated using Illumina GenomeStudioGT v. 1.8.4 software were used to generate PLINK input files (that is, Igen, map and fam files). We used the MDS analysis of genome-wide identity-by-state data implemented in PLINK to determine ancestry in the whole sample. The analysis was conducted using the 688890 SNPs that passed quality control filters previously described. The first two components from the MDS analysis identified clearly separated clusters that correlate with self-reported ethnicity identification. The first two MDS components distinguished African American from European American participants and others and the second component distinguished Hispanic and non-Hispanic subjects. To reduce population stratification, we removed all individuals who described themselves as White/Caucasian in the association analyses $(n=7)$. Both components were used to adjust for any remaining population stratification in the association analyses.

Statistical analyses. Analyses were conducted on the 399 individuals who had each (1) smoked cigarettes (on at least some days) in their lifetime, (2) had data available on cigarette use and (3) consented to the collection of DNA. Only individuals with complete data available were included in the analyses. For the analytic sample $(n=399)$, descriptive statistics on key variables, as well as a comparison to the full sample is provided in Table 1.

Polygenic risk score. First, we constructed a polygenic risk score in which we combined the top genetic variants (all SNPs reaching a $P$-value threshold of $5 \times 10^{-7}$ ) described in the STOMP meta-analysis. ${ }^{18}$ We limited the polygenic risk score to include only variants associated with the number of cigarettes smoked per day (and not age of initiation, which was also analyzed in the STOMP meta-analysis) to limit phenotypic heterogeneity. This included six SNPs from multiple genes across chromosomes 15 and 1 (detailed in Table 2). SNPs were in modest linkage disequilibrium ( $r^{2}$ values ranged from 0.07 to 0.47 ), limiting the concern of redundancy in the genetic risk score (GRS). In cases where the original SNP was not available in the DNHS, a highly correlated $\left(r^{2}>0.9\right)$ proxy SNP was used in its place. Each of the SNPs was weighted by the marker information (risk allele and effect size) provided from the meta-analysis; each variant (risk allele) was summed to create an aggregate GRS, and those variants with a larger effect size (indicated by the $\beta$-coefficient) were given a greater weight. Scores were computed using PLINK. ${ }^{45}$

Main effects. Once GRSs were computed for each individual, we examined whether the GRS was associated with frequency of cigarettes smoked per day. Next, we examined the relationship between aspects of the individual's social environment, including the experience of traumatic life events, neighborhood social cohesion and neighborhood physical disorder, and whether each environmental variable was associated with the number of cigarettes smoked per day in this population. Because of the skewed distribution of cigarettes smoked per day, all analyses were conducted using Poisson regression in SAS (SAS Institute, 2003), adjusting for sex, age and ancestral components (described previously), as well as appropriate sample weights.

Gene-environment correlation and interaction. Next, we tested for association between the GRS and the experience of traumatic life events, neighborhood social cohesion and neighborhood physical disorder. That is, we conducted a test of Spearman's rGE in SAS (SAS Institute, 2003), as the presence of rGE undermines the interpretation of GxE. ${ }^{46} \mathrm{We}$ then examined how the GRS interacted with environmental exposures, for which we did not find a significant rGE to predict cigarette smoking in this population. Additive interactions were tested in SAS (SAS Institute, 2003), using sex, age and ancestry as covariates. We assessed the interaction between the GRS and each environmental variable on the additive scale by including a cross-product term in separate linear-Poisson regression models. The estimate for the cross-product term represents the interaction contrast (IC), that is, the difference in the differences of the mean number of cigarettes smoked per day when the risky environment (increased number of traumatic events, decreased neighborhood cohesion and increased neighborhood physical disorder) is present versus absent for those with and without increased GRSs. $\chi^{2}$ tests were used to assess the statistical significance of the IC and mean differences (MDs) at various levels of exposure. Plots of regression-adjusted mean phenotypes with $95 \%$ confidence intervals are presented to 
Table 2 Top genetic variants associated with cigarettes per day in the STOMP Consortium $\left(P\right.$-value $<5 \times 10^{-7}$; David et al. $\left.{ }^{18}\right)$ included in the Detroit Neighborhood Health Study Genetic Risk Score $(n=399)$

\begin{tabular}{|c|c|c|c|c|c|c|}
\hline SNP & Chromosome & BP position & Nearby genes & Alleles & Coded AF & Effect size \\
\hline rs2036527 & 15 & 76638670 & CHRNA5 & $\mathrm{A} / \mathrm{G}$ & 0.22 & 0.040 \\
\hline rs667282 & 15 & 76650527 & CHRNA5 & $\mathrm{C} / \mathrm{T}$ & 0.29 & 0.033 \\
\hline rs3101457 & 1 & 242599837 & C1orf100 & $\mathrm{A} / \mathrm{G}$ & 0.75 & 0.041 \\
\hline rs938682 & 15 & 76683602 & CHRNA3 & $\mathrm{A} / \mathrm{G}$ & 0.71 & 0.033 \\
\hline rs547843 & 15 & 23975140 & LOC503519 & $\mathrm{C} / \mathrm{G}$ & 0.65 & -0.035 \\
\hline rs3813570 ${ }^{a}$ & 15 & 76619887 & PSMA4 & $\mathrm{C} / \mathrm{T}$ & 0.26 & 0.033 \\
\hline
\end{tabular}

Abbreviations: AF, allele frequency; BP, base pair position; CHRNA5=cholinergic receptor, nicotinic, alpha 5 ; $\mathrm{C} 1$ orf100 = uncharacterized chromosome 1 open reading frame; CHRNA3 = cholinergic receptor, nicotinic, alpha 3; LOC503519= uncharacterized; PSMA4=proteasome (prosome, macropain) subunit, alpha type 4; SNP, single-nucleotide polymorphism.

${ }^{a}$ Note: When the original STOMP (David et al. ${ }^{18}$ ) SNP was not available for analysis in the DNHS, a proxy SNP $\left(r^{2}>0.9\right)$ was used in its place; rs667282 served as a proxy for rs667282 $\left(r^{2}=0.924\right)$, rs7163730 served as a proxy for rs3813570 $\left(r^{2}=0.916\right)$.
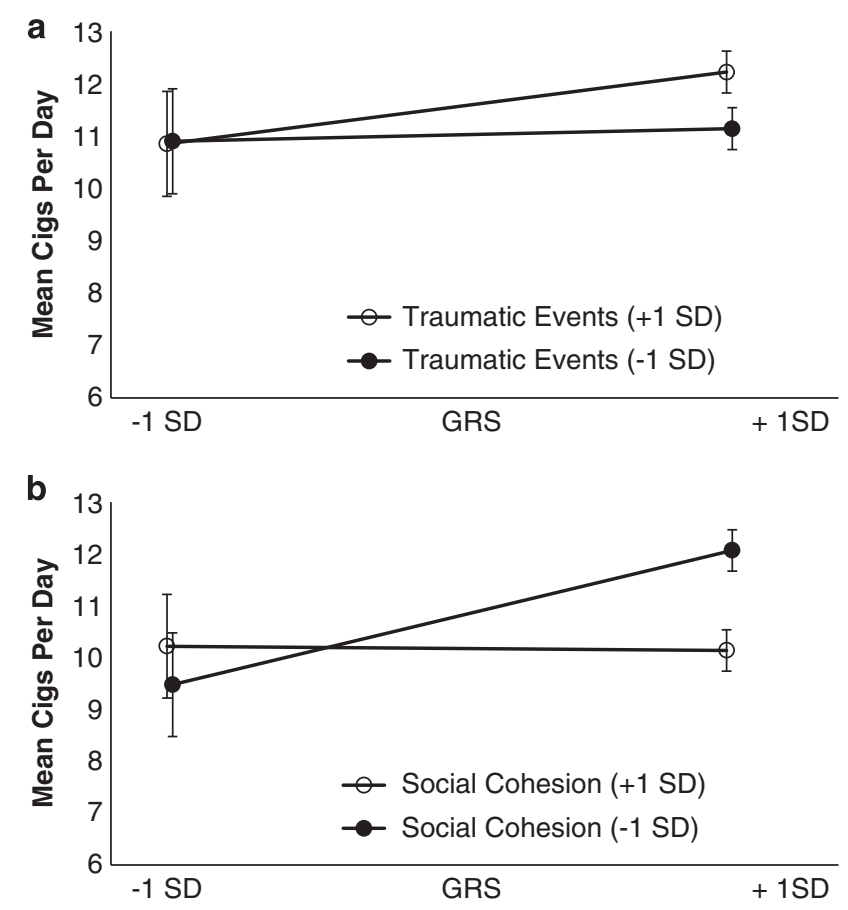

Figure 1 The Interaction between genetic and environmental predictors of cigarette use in 399 individuals from the Detroit Neighborhood Health Study. (a) Genetic risk for smoking (GRS +1 s.d.) was greater for individuals who had experienced an increased number of traumatic events in their lifetimes. (b) Genetic risk for smoking (GRS +1 s.d.) was greater for individuals who lived in a neighborhood characterized by less social cohesion.

facilitate interpretation. Note that Table 4 and Figure 1 present results for each environmental exposure as decreased (1 s.d. below the mean ( -1 s.d.)) and increased (1 s.d. above the mean $(+1 S D)$ ). Similarly, an increased GRS is defined as +1 s.d. and a decreased GRS is defined as -1 s.d. As tests of GxE are often sensitive to scale, ${ }^{47}$ we standardized all variables ( $z$-score) to ensure that results remained robust to transformations.

\section{Results}

Descriptive statistics. Over half of the sample $(n=406$, $52.2 \%$ ) reported having smoked cigarettes on at least some days in their lifetime (former smokers included). For these
Table 3 Main effects of (a) genetic risk score, (c) traumatic events, (d) neighborhood social cohesion and (e) neighborhood physical disorder on cigarette use (cigarettes per day) in the Detroit Neighborhood Health Study $(n=399)$

\begin{tabular}{lccc}
\hline & \multicolumn{3}{c}{ Cigarettes per day } \\
\cline { 2 - 4 } Predictor & Risk ratio $(95 \%$ Cl) & P-value & $r^{2}$ \\
\hline b. Genetic risk score & $1.76(0.89,3.49)$ & 0.04 & 0.03 \\
c. Traumatic events & $1.09(0.46,2.60)$ & 0.01 & 0.01 \\
d. Social cohesion & $0.86(0.68,1.09)$ & 0.02 & 0.01 \\
e. Physical disorder & $0.56(0.47,0.66)$ & 0.03 & 0.01
\end{tabular}

Abbreviation: $\mathrm{Cl}$, confidence interval.

$r^{2}$ refers to the proportion of total variance in cigarettes per day accounted by each predictor.

${ }^{a}$ Adjusted for age, sex and ancestry using overdispersed Poisson regression.

individuals, the number of cigarettes smoked per day ranged from 0 to 98 cigarettes (mean $=11.16$, s.d. $=12.19$ ) in the last 30 days. The number of lifetime traumatic events reported in this sample ranged from 0 to 19 (mean $=5.87$, s.d.=3.87). Scores indicating the sense of neighborhood cohesion in this sample ranged from 0 to 16 (mean $=3.41$, s.d.=1.48). Factor scores indicating the level of neighborhood physical order/disorder in this sample ranged from -2.87 to $3.76($ mean $=-0.10$, s.d. $=0.69)$. Polygenic risk scores ranged from -0.01 to 0.02 (mean $=0.01$, s.d. $=0.01$; distribution is depicted in Supplementary Figure 2).

Main effects. Among the analytic sample, the GRS predicted number of cigarettes smoked per day (risk ratio $=1.76$ $(0.89,3.49))$ and accounted for $\sim 3 \%$ of the overall variance in this trait. Traumatic events, neighborhood social cohesion and physical disorder were related to cigarette use (risk ratio $=1.09(0.46,2.60), 0.86(0.68,1.09)$ and $0.56(0.47$, $0.66)$, respectively). Moreover, whereas traumatic events and neighborhood physical disorder are associated with an increase in the number of cigarettes smoked per day, neighborhood social cohesion is associated with fewer cigarettes smoked per day. All results are detailed in Table 3.

Gene-environment correlation and interaction. We found evidence of rGE between the GRS and neighborhood physical disorder $\left(r^{2}=0.14, P<0.01\right)$, but no significant correlation was observed between the GRS and neighborhood social cohesion or number of traumatic events. Due to 
Table 4 Additive effects of (a) traumatic events, (b) neighborhood social cohesion and genetic risk on cigarette use in the Detroit Neighborhood Health Study

\begin{tabular}{|c|c|c|}
\hline Environmental exposure & Genetic risk score & $\begin{array}{l}\text { Cigarettes per day } \\
\text { mean }(95 \% \mathrm{Cl})^{\mathrm{a}}\end{array}$ \\
\hline \multicolumn{3}{|l|}{ Traumatic life events } \\
\hline-1 s.d. $(n=685)$ & -1 s.d. $(n=195)$ & $\begin{array}{l}10.91(09.94,11.89) \\
11.22(10.44 .11 .29)\end{array}$ \\
\hline Difference $^{\mathrm{b}}$ & & $0.31(-0.50,0.60)$ \\
\hline+1 s.d. $(n=862)$ & -1 s.d. $(n=295)$ & $10.98(09.97,11.79)$ \\
\hline Difference ${ }^{b}$ & +1 s.d. $(n=102)$ & $\begin{array}{l}12.20(11.58,12.52) \\
1.22^{\mathrm{c}}(0.73,1.61)\end{array}$ \\
\hline Interaction contrast ${ }^{\mathrm{C}}$ & & $0.91^{\mathrm{cd}}(0.23,1.01)$ \\
\hline \multicolumn{3}{|c|}{ Neighborhood social cohesion } \\
\hline-1 s.d. $(n=520)$ & $\begin{array}{l}-1 \text { s.d. }(n=171) \\
+1 \text { s.d. }(n=57)\end{array}$ & $\begin{array}{l}09.48(08.80,10.56) \\
12.07(11.88,12.26)\end{array}$ \\
\hline Difference $^{b}$ & & $2.59^{\mathrm{C}}(1.70,3.08)$ \\
\hline+1 s.d. $(n=1011)$ & -1 s.d. $(n=317)$ & $10.14(9.44,11.23)$ \\
\hline & + 1 s.d. $(n=102)$ & $10.22(9.47,10.18)$ \\
\hline $\begin{array}{l}\text { Difference } \\
\text { Interaction contrast }^{\mathrm{d}}\end{array}$ & & $\begin{aligned} & 0.08(-1.85,2.03) \\
& 2.51^{\mathrm{c}}(1.05,3.55)\end{aligned}$ \\
\hline
\end{tabular}

aAdjusted for age, sex and ancestry using overdispersed Poisson regression. ${ }^{b}$ Difference between the mean for presence of high genetic risk versus low genetic risk, calculated on the mean (additive) scale using overdispersed Poisson regression with linear link function. ${ }^{c} P$-value $<0.05$. ${ }^{d}$ Difference of the differences; additive interaction is indicated when the difference between the mean differences for the environmental exposure group versus the noexposure group is significantly $>0$.

the significant rGE observed between the GRS and neighborhood physical disorder, we did not test for GxE with this social environment.

Significant additive interactions were observed between the GRS and the experience of traumatic events and neighborhood social cohesion. The association between genetic risk and smoking was greater for individuals who had experienced traumatic events in their lifetimes, and diminished for individuals who had increased social cohesion in their neighborhood (depicted in Figure 1). Moreover, higher mean numbers of cigarettes smoked per day were indicated for those with increased ( +1 s.d.) genetic risk in both than in those who experienced a greater $(+1$ s.d.) number of traumatic events (MD between high ( +1 s.d.) and low ( -1 s.d.) GRS =1.22) and those who had experienced a decreased ( -1 s.d.) number of events $(M D=0.31)$, but the genetic influence was even greater for those who experienced an increased (+1s.d.) number of traumatic events $(\mathrm{IC}=0.91, P<0.05$; detailed in Table 4, depicted in Figure 1a). Similarly, higher mean numbers of cigarettes smoked per day were indicated for those with increased ( +1 s.d.) genetic risk in neighborhoods characterized by diminished ( -1 s.d.) social cohesion (MD between high and low GRS = 2.59) and in neighborhoods characterized by greater ( +1 s.d.) social cohesion $(M D=0.08)$; however, the genetic influence was even greater for those who lived in neighborhoods characterized by less ( -1 s.d.) social cohesion $(\mathrm{IC}=2.51, P<0.05$; detailed in Table 4, depicted in Figure $1 \mathrm{~b})$.

\section{Conclusions}

In this study, we found that a polygenic risk score, created by using genetic variants from the STOMP meta-analysis, predicted the frequency of cigarettes smoked per day in a representative sample of African American adult residents of
Detroit. In addition, we found that features of the neighborhood social and physical environments, including social cohesion and physical disorder, were associated with cigarette use in this population. Finally, we found the evidence of gene-environment interaction; the association between genetic risk and smoking was greater for individuals who had experienced trauma in their lifetimes and diminished for individuals who had increased social cohesion in their neighborhoods. These results agree with previous studies suggesting that riskier environments (for example, increased stressful life events and fewer social constraints) allow for an increased expression of genetic predispositions towards substance use. ${ }^{39}$ In using the aggregate measure of genetic risk, informed by the previous literature, we move geneenvironment interaction studies forward by more accurately modeling the genetic architecture of cigarette smoking.

The relationship between polygenic risk and cigarette use. The polygenic risk score, comprised of the top genetic variants $\left(P\right.$-value $<5 \times 10^{-7}$ ) from the STOMP replicated in the DNHS, accounted for $\sim 3 \%$ of the total variance in cigarette-use frequency. In the current literature, polygenic risk scores typically account for $0.5-4 \%$ of the variance in a complex behavioral trait. ${ }^{7,8}$ Post hoc analyses indicated that the effect of the polygenic score was largely driven by the effect of CHRNA5 SNP rs203652, the only genetic variant reaching genome-wide significance in the STOMP; however, the addition of the five other variants significantly increased the total variance $\sim 0.8 \%$ in smoking accounted for the GRS (Supplementary Table 1). There are growing numbers of studies that have found an association between a variant in CHRNA5 and smoking phenotypes in diverse populations. ${ }^{48}$ However, this SNP accounted for $\sim 0.2 \%$ of the phenotypic variance of CPD in the African American STOMP meta-analysis, while a correlated SNP (rs1051730 A allele) accounted for $0.5 \%$ of the phenotypic variance in smoking quantity in populations of European ancestry. ${ }^{18}$ Evidence from animal and magnetic resonance imaging studies support these findings; a study of CHRNA5 knockout mice showed that reexpressing this gene in the medial habenula, which extends projections to a brain region shown to mediate nicotine withdrawal, abolished the inhibitory effects of nicotine while maintaining the reinforcing effects of nicotine. ${ }^{49,50}$ In a functional magnetic resonance imaging study of smokers, genetic variation in CHRNA5 appeared to also affect the reactivity to smoking cues in the insula, hippocampus and dorsal striatum, regions implicated in addictive behavior and memory. ${ }^{51}$ Thus, it is biologically plausible that increased expression of CHRNA5 could be associated with smoking quantity as a consequence of neuro-adaptations resulting from complex interactions between genes and environment.

The GRS was not related to traumatic events or social cohesion, limiting the concern that gene-environment correlation may bias gene-environment interaction results (type I error). The GRS was, however, related to neighborhood physical disorder; greater genetic risk for cigarette-use frequency was associated with living in a neighborhood characterized by increased physical disorder. This may indicate that there are shared risk factors for cigarette use and neighborhood quality. 
The relationship between social risk factors and cigarette use. Our study suggests that in Detroit, neighborhood social cohesion and physical disorder have opposite associations with cigarette smoking among African Americans. These findings are consistent with prior research. In a recent study, ${ }^{24}$ the relationship between social cohesion and smoking was examined in Black, treatment-seeking, smokers. They found that, although the total effect of social cohesion on continuous abstinence was nonsignificant, social cohesion was associated with social support, positive affect, negative affect and stress, which, in turn, were each associated with abstinence. In another study of smoking behaviors in urban neighborhoods, higher collective efficacy, which represents a combination of social cohesion and social control, was associated with more smoking in neighborhoods where smoking norms were permissive; in contrast, higher collective efficacy was associated with less smoking in neighborhoods where norms were strongly antismoking. ${ }^{47}$ Taken together, these results suggest that social cohesion may facilitate decreased smoking through psychosocial mechanisms that can result from living in a community with strong interpersonal connections and antismoking values; however, this effect is likely contingent upon social norms within the neighborhood. ${ }^{52}$ Although previous studies have examined several physical aspects of the neighborhood in relation to health outcomes, including depression ${ }^{53}$ and substance use, ${ }^{27}$ no study to our knowledge has explicitly examined the relationship between neighborhood physical disorder and cigarette-smoking frequency. Studies that have examined the relationship between physical disorder and mental health outcomes found that poorer neighborhood conditions (that is, more physical disorders) were related to worse outcomes (that is, more depressive or alcohol use disorder symptoms). The direction of effect was similar for this study's finding in that poorer neighborhood conditions (more physical disorders) were associated with a greater frequency of cigarettes smoked. One key mechanism underlying this relationship has been posited; the stressreduction hypothesis suggests that substance use may be used to relieve distress. ${ }^{54}$ Therefore, individuals living in physically deteriorated neighborhoods may use cigarette smoking as a coping mechanism in response to the stressful life experiences that cluster in neighborhoods with high levels of physical disorder.

The relationship between polygenic and environmental risk factors and cigarette use. In the DNHS, we found support for a significant additive interaction between the GRS and traumatic events that was associated with the frequency of cigarette smoking. As mentioned above, several previous studies have reported an interaction between a specific genetic variant and some aspects of trauma as a predictor of cigarette smoking. ${ }^{32,35}$ Although genetic risk was related to an increase in the number of cigarettes smoked per day, the influence of the genetic risk variants for smoking was greater for individuals who had experienced trauma in their lifetimes than for individuals who had not. In this study, we expanded upon these results by examining the interaction between the experience of traumatic events and an aggregate measure of molecular genetic risk for cigarette smoking. The association between the GRS and cigarette smoking was stronger for individuals who had experienced more traumatic events.

We also found support for a significant additive interaction between the GRS and neighborhood social cohesion that was associated with frequency of cigarette smoking. Genetic risk for smoking was less influential for individuals who lived in neighborhoods with higher levels of social cohesion. To our knowledge, no previous study has specifically examined the relationship between genetic risk factors and neighborhood social cohesion, as measured here. However, there have been studies that have examined the interaction between genetic risk factors and related environmental risk factors, such as rural residencies. ${ }^{25,41}$ In these studies, environmental contexts characterized by accountability to your peers, neighbors and community diminished the genetic influences on substance use. We believe this to be an example of social control, ${ }^{39}$ whereby aspects of the social environment restrict the opportunity to express one's genetic predisposition for a risk behavior (cigarette smoking). These results, taken together with previous studies that have found an inverse relationship between social support and substance use in adolescents, ${ }^{26}$ suggest that these supportive relationships may act as protective factors against the risk of smoking behaviors. However, in light of previous studies that have examined the mechanism behind neighborhood social cohesion, ${ }^{52}$ this is likely contingent upon neighborhood antismoking attitudes.

There are several strengths of this study. We replicated common genetic variation in a sample, which also includes detailed environmental information. In addition, we used a relatively novel polygene score-environment interaction method, which allowed a focus on the social context in this study. We examined one commonly studied social context (traumatic life events) and two novel aspects of the individual's neighborhood (social cohesion and physical disorder). Most importantly, this is a unique sample collected for the study of genetic and environmental influences on mental health and related traits in a population primarily of non-European ancestry.

There are several limitations of this study as well. First, because of the sample size, this study is limited in the potential strength of the association signal. A power calculation indicated $78 \%$ power to detect the main effects, given the parameters of this study. However, the rich environmental data available on this sample provide the opportunity to refine genetic associations discovered and validated in larger, better powered samples. Second, this polygenic risk score is largely driven by a single variant (CHRNA5 SNP rs203652) and therefore is not truly polygenic in nature. However, predictive power is gained from the inclusion of the other genetic variants in the risk score. Finally, the present analysis uses data available at a single time point, using retrospective reports of smoking, traumatic life events and neighborhood characteristics.

Although the genetic and environmental influences on cigarette-smoking behavior have been well established, translation of this research into useful risk-factor targets for public health intervention is lacking. Among the reasons is the complex nature of smoking behaviors, which are influenced by multiple genetic and environmental risk factors, each having very subtle effects. Related is the limitation of current 
statistical and genetic methodologies to successfully identify novel, replicable variants in relatively small $(n<1,000)$ samples. Despite these limitations, evidence for the influence of gene-environment interactions on smoking behaviors is mounting, both from molecular genetic studies and epidemiologic studies. Polygenic risk scores are an aggregate measure of genetic variants that influence the trait of interest and therefore do not require the rigorous statistical test corrections required for GWAS. Importantly, these methods allow for the examination of specific environmental risk factors and how genetic influences may change as a function of the environmental risk factor. These risk factors, and how they can diminish or exacerbate genetic influences on cigarette use, have important implications for public health interventions as they are potentially modifiable risk factors.

In conclusion, we modeled the genetic architecture of smoking behaviors in African Americans living in Detroit. We constructed a GRS that predicted cigarettes smoked per day and accounted for $\sim 3 \%$ of the overall variance in the trait. In addition, significant additive interactions were observed between this GRS and aspects of the individual's social context, including the experience of traumatic events and neighborhood social cohesion. This study provides support for the utility of the GRS as an alternative approach to the replication of common polygenic variation, and geneenvironment interaction, in smoking behaviors. In addition, this study suggests that interventions should target individuals who have experienced traumatic life events and emphasizes the potential importance that promotion of neighborhood-level social cohesion may have in diminishing genetic vulnerability to smoking behaviors.

\section{Conflict of interest}

The authors declare no conflict of interest.

Acknowledgements. The DNHS was approved by the Institutional Review Board at the University of Michigan (HUM00014138; FWA00004969 and OHRP IRB IRB00000245).

1. Oza S, Thun MJ, Henley SJ, Lopez AD, Ezzati M. How many deaths are attributable to smoking in the United States? Comparison of methods for estimating smoking-attributable mortality when smoking prevalence changes. Prev Med 2011; 52: 428-433.

2. Broms U, Silventoinen K, Madden PAF, Heath AC, Kaprio J. Genetic architecture of smoking behavior: a study of Finnish adult twins. Twin Res Hum Genet 2006; 9: 64-72.

3. Lessov CN, Martin NG, Statham DJ, Todorov AA, Slutske WS, Bucholz KK et al. Defining nicotine dependence for genetic research: evidence from Australian twins. Psychol Med 2004; 34: 865-879.

4. Thorgeirsson TE, Geller F, Sulem P, Rafnar T, Wiste A, Magnusson KP et al. A variant associated with nicotine dependence, lung cancer and peripheral arterial disease. Nature 2008; 452: 638-642.

5. Liu JZ, Tozzi F, Waterworth DM, Pillai SG, Muglia P, Middleton L et al. Meta-analysis and imputation refines the association of 15q25 with smoking quantity. Nat Genet 2010; 42 : 436-440.

6. Furberg H, Kim Y, Dackor J, Boerwinkle E, Franceschini N, Ardissino D et al. Genome-wide meta-analyses identify multiple loci associated with smoking behavior. Nat Genet 2010; 42 : 441-447.

7. Aulchenko YS, Struchalin MV, Belonogova NM, Axenovich TI, Weedon MN, Hofman A et al. Predicting human height by Victorian and genomic methods. Eur J Hum Genet 2009; 17: 1070-1075.

8. Purcell SM, Wray NR, Stone JL, Visscher PM, O'Donovan MC, Sullivan PF et al. Common polygenic variation contributes to risk of schizophrenia and bipolar disorder. Nature 2009; 460: $748-752$.
9. Boardman JD, Saint Onge JM, Haberstick BC, Timberlake DS, Hewitt JK. Do schools moderate the genetic determinants of smoking? Behav Genet 2008; 38: 234-246.

10. Boardman JD. State-level moderation of genetic tendencies to smoke. Am J Public Health 2009 (cited 2013) 99: 480-486.

11. Daw J, Shanahan M, Harris KM, Smolen A, Haberstick B, Boardman JD. Genetic sensitivity to peer behaviors: 5HTTLPR, smoking, and alcohol consumption. J Health Soc Behav 2013 (cited 2013) 54: 92-108.

12. Boardman JD, Alexander KB, Stallings MC. Stressful life events and depression among adolescent twin pairs. Biodemography Soc Biol 2011 (cited 2013) 57: 53-66.

13. Peterson RE, Maes HH, Holmans P, Sanders AR, Levinson DF, Shi J et al. Genetic risk sum score comprised of common polygenic variation is associated with body mass index. Hum Genet 2011 (cited 2013) 129: 221-230.

14. Belsky DW, Moffitt TE, Baker TB, Biddle AK, Evans JP, Harrington $\mathrm{H}$ et al. Polygenic risk and the developmental progression to heavy, persistent smoking and nicotine dependence: evidence from a 4-decade longitudinal study. JAMA Psychiatry 2013 (cited 2013) 70: 534-542.

15. Uhl GR, Walther D, Musci R, Fisher C, Anthony JC, Storr CL et al. Smoking quit success genotype score predicts quit success and distinct patterns of developmental involvement with common addictive substances. Mol Psychiatry advance online publication, 6 November 2012; doi:10.1038/mp.2012.155 (e-pub ahead of print).

16. Rosenberg NA, Huang L, Jewett EM, Szpiech ZA, Jankovic I, Boehnke M. Genome-wide association studies in diverse populations. Nat Rev Genet 2010; 11: 356-366.

17. Bierut LJ. Convergence of genetic findings for nicotine dependence and smoking related diseases with chromosome 15q24-25. Trends Pharmacol Sci 2010; 31: 46-51.

18. David SP, Hamidovic A, Chen GK, Bergen AW, Wessel J, Kasberger JL et al. Genomewide meta-analyses of smoking behaviors in African Americans. Transl Psychiatry 2012; 2: e119-e119.

19. Little HJ. Behavioral mechanisms underlying the link between smoking and drinking Alcohol Res Health 2000 (cited 2013) 24: 215-224.

20. Novotny TE, Warner KE, Kendrick JS, Remington PL. Smoking by blacks and whites: socioeconomic and demographic differences. Am J Public Health 1988 (cited 2013) 78: 1187-1189.

21. Gundersen DA, Delnevo CD, Wackowski O. Exploring the relationship between race/ ethnicity, menthol smoking, and cessation, in a nationally representative sample of adults. Prev Med[Internet] 2009 (cited 2013) 49: 553-557.

22. Haiman CA, Stram DO, Wilkens LR, Pike MC, Kolonel LN, Henderson BE et al. Ethnic and racial differences in the smoking-related risk of lung cancer. N Engl J Med 2006; 354: 333-342.

23. Roberts ME, Fuemmeler BF, McClernon FJ, Beckham JC. Association between trauma exposure and smoking in a population-based sample of young adults. $J$ Adolesc Health 2008; 42: 266-274.

24. Reitzel LR, Businelle MS, Kendzor DE, Li Y, Cao Y, Castro Y et al. Subjective social status predicts long-term smoking abstinence. BMC Public Health 2011; 11: 135.

25. Galea S, Vlahov D. Social determinants and the health of drug users: socioeconomic status, homelessness, and incarceration. Public Health Rep 2002; 117: S135-S145.

26. Lin E-Y, Witten K, Casswell S, You RQ. Neighbourhood matters: perceptions of neighbourhood cohesiveness and associations with alcohol, cannabis and tobacco use. Drug Alcohol Rev 2012; 31: 402-412.

27. Cerdá M, Diez-Roux AV, Tchetgen ET, Gordon-Larsen P, Kiefe C. The relationship between neighborhood poverty and alcohol use: estimation by marginal structural models. Epidemiology 2010; 21: 482-489.

28. Keyes KM, McLaughlin KA, Koenen KC, Goldmann E, Uddin M, Galea S. Child maltreatment increases sensitivity to adverse social contexts: neighborhood physical disorder and incident binge drinking in Detroit. Drug Alcohol Depend 2012; 122: 77-85.

29. Lopez-Quintero C, Pérez de los Cobos J, Hasin DS, Okuda M, Wang S, Grant BF et al. Probability and predictors of transition from first use to dependence on nicotine, alcohol, cannabis, and cocaine: results of the National Epidemiologic Survey on Alcohol and Related Conditions (NESARC). Drug Alcohol Depend 2011 (cited 2013) 115: 120-130.

30. Lerer E, Kanyas K, Karni O, Ebstein RP, Lerer B. Why do young women smoke? II. Role of traumatic life experience, psychological characteristics and serotonergic genes. $\mathrm{Mol}$ Psychiatry 2006; 11: 771-781.

31. Mingione CJ, Heffner JL, Blom TJ, Anthenelli RM. Childhood adversity, serotonin transporter (5-HTTLPR) genotype, and risk for cigarette smoking and nicotine dependence in alcohol dependent adults. Drug Alcohol Depend 2011; 123: 201-206.

32. Segman RH, Kanyas K, Karni O, Lerer E, Goltser-Dubner T, Pavlov V et al. Why do young women smoke? IV. Role of genetic variation in the dopamine transporter and lifetime traumatic experience. Am J Med Genet 2007; 144B: 533-540.

33. Greenbaum L, Kanyas K, Karni O, Merbl Y, Olender T, Horowitz A et al. Why do young women smoke? I. Direct and interactive effects of environment, psychological characteristics and nicotinic cholinergic receptor genes. Mol Psychiatry 2006; 7: 223.

34. Xie P, Kranzler HR, Zhang H, Oslin D, Anton RF, Farrer LA et al. Childhood adversity increases risk for nicotine dependence and interacts with $\alpha 5$ nicotinic acetylcholine receptor genotype specifically in males. Neuropsychopharmacology 2012 (cited 2013) 37: 669-676. 
35. Nilsson KW, Sjöberg RL, Damberg M, Alm PO, Ohrvik J, Leppert J et al. Role of the serotonin transporter gene and family function in adolescent alcohol consumption.. Alcohol Clin Exp Res 2005; 29: 564-570.

36. Rose RJ, Dick DM, Viken And RJ, Kaprio J. Gene-environment interaction in patterns of adolescent drinking: regional residency moderates longitudinal influences on alcohol useAlcohol Clin Exp Res 2001; 25: 637-643.

37. Dick DM, Rose RJ, Viken RJ, Kaprio J, Koskenvuo M. Exploring gene-environment interactions: socioregional moderation of alcohol use. J Abnorm Psychol 2001; 110 625-632.

38. Dick DM, Bernard M, Aliev F, Viken R, Pulkkinen L, Kaprio J et al. The role of socioregional factors in moderating genetic influences on early adolescent behavior problems and alcohol use. Alcohol Clin Exp Res 2009; 33: 1739-1748.

39. Shanahan MJ, Hofer SM. Social context in gene-environment interactions: retrospect and prospect. J Gerontol B Psychol Sci Soc Sci 2005; 60 Spec No: 65-76.

40. Uddin M, Aiello AE, Wildman DE, Koenen KC, Pawelec G, De Los Santos R et al. Epigenetic and immune function profiles associated with posttraumatic stress disorder Proc Natl Acad Sci USA 2010; 107: 9470-9475.

41. Sampson RJ, Raudenbush SW, Earls F. Neighborhoods and violent crime: a multilevel study of collective efficacy. Science (New York, NY) 1997 (cited 2013) 277: 918-924.

42. Johns LE, Aiello AE, Cheng C, Galea S, Koenen KC, Uddin M. Neighborhood socia cohesion and posttraumatic stress disorder in a community-based sample: findings from the Detroit Neighborhood Health Study. Soc Psychiatry Psychiatr Epidemiol 2012 (cited 2013) 47: 1899-1906.

43. Ompad DC, Fuller CM, Chan CA, Frye V, Vlahov D, Galea S. Correlates of illicit methadone use in New York City: A cross-sectional study. BMC Public Health 2008; 8: 375.

44. Logue MW, Baldwin C, Guffanti G, Melista E, Wolf EJ, Reardon AF et al. A genome-wide association study of post-traumatic stress disorder identifies the retinoid-related orphan receptor alpha (RORA) gene as a significant risk locus. Mol psychiatry advance online publication, 7 August 2012; doi:10.1038/mp.2012.113 (e-pub ahead of print).

45. Purcell S, Neale B, Todd-Brown K, Thomas L, Ferreira MAR, Bender D et al. PLINK: a too set for whole-genome association and population-based linkage analyses. Am J Hum Genet 2007; 81: 559-575.
46. Moffitt TE, Caspi A, Rutter M. Strategy for investigating interactions between measured genes and measured environments. Arch Gen Psychiatry 2005; 62: 473-481.

47. Eaves LJ. Genotype x Environment interaction in psychopathology: fact or artifact? Twin res hum genet 2006 (cited 2013) 9: 1-8.

48. Chen L-S, Saccone NL, Culverhouse RC, Bracci PM, Chen C-H, Dueker N et al. Smoking and genetic risk variation across populations of European, Asian, and African American ancestry-a meta-analysis of chromosome 15q25. Genet Epidemiol 2012; 36: 340-351.

49. Salas R, Sturm R, Boulter J, De Biasi M. Nicotinic receptors in the habenulointerpeduncular system are necessary for nicotine withdrawal in mice. J Neurosci 2009; 29 : 3014-3018.

50. Fowler CD, Lu Q, Johnson PM, Marks MJ, Kenny PJ. Habenular $\alpha 5$ nicotinic receptor subunit signalling controls nicotine intake. Nature 2011; 471: 597-601.

51. Janes AC, Smoller JW, David SP, Frederick BD, Haddad S, Basu A et al. Association between CHRNA5 genetic variation at rs 16969968 and brain reactivity to smoking images in nicotine dependent women. Drug Alcohol Depend 2012; 120: 7-13.

52. Ahern J, Galea S, Hubbard A, Syme SL. Neighborhood smoking norms modify the relation between collective efficacy and smoking behavior. Drug Alcohol Depend 2009; 100: 138-145.

53. Beard JR, Cerdá M, Blaney S, Ahern J, Vlahov D, Galea S. Neighborhood characteristics and change in depressive symptoms among older residents of New York city. Am J Public Health 2009; 99: 1308-1314.

54. Sinha R. How does stress increase risk of drug abuse and relapse? Psychopharmacology 2001; 158: 343-359.

(c) (i) (3) $\odot$ Translational Psychiatry is an open-access journal published by Nature Publishing Group. This work is licensed under a Creative Commons Attribution-NonCommercialNoDerivs 3.0 Unported License. To view a copy of this license, visit http://creativecommons.org/licenses/by-nc-nd/3.0/

Supplementary Information accompanies the paper on the Translational Psychiatry website (http://www.nature.com/tp) 